
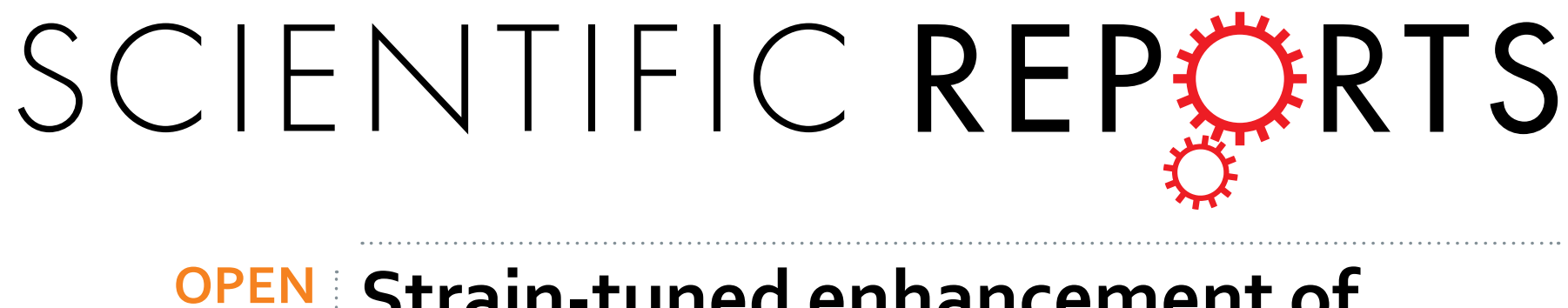

\title{
Strain-tuned enhancement of
} ferromagnetic $T_{C}$ to $176 \mathrm{~K}$ in Sm-doped $\mathrm{BiMnO}_{3}$ thin films

Received: 10 November 2016

Accepted: 30 January 2017

Published: 03 March 2017

\section{and determination of magnetic phase diagram}

\author{
Eun-Mi Choi, Josée E. Kleibeuker \& Judith L. MacManus-Driscoll
}

$\mathrm{BiMnO}_{3}$ is a promising multiferroic material but it's ferromagnetic $T_{C}$ is well below room temperature and the magnetic phase diagram is unknown. In this work, the relationship between magnetic transition temperature $\left(T_{C}\right)$ and the substrate induced (pseudo-) tetragonal distortion (ratio of outof-plane to in-plane lattice parameters, $c / a$ ) in $\mathrm{BiMnO}_{3}$ thin films, lightly doped to optimize lattice dimensions, was determined. For $c / a>0.99$, hidden antiferromagnetism was revealed and the magnetisation versus temperature curves showed a tail behaviour, whereas for $c / a<0.99$ clear ferromagnetism was observed. A peak $T_{C}$ of up to $176 \mathrm{~K}$, more than $70 \mathrm{~K}$ higher than for bulk $\mathrm{BiMnO}_{3}$, was achieved through precise strain tuning. The $T_{C}$ was maximised for strong tensile in-plane strain which produced weak octahedral rotations in the out-of-plane direction, an orthorhombic-like structure, and strong ferromagnetic coupling.

Strong coupling between magnetism and ferroelectricity in perovskite oxides $\left(\mathrm{ABO}_{3}\right)$ has drawn increasing interest because of the potential for magnetoelectric devices for highly sensitive magnetic sensors and low energy random access memory ( $\mathrm{RAM}) . \mathrm{BiMnO}_{3}(\mathrm{BMO})$ has been extensively investigated because it is one of the rare multiferroics that possesses both ferromagnetism (FM) and ferroelectricity (FE). However, its maximum reported magnetic transition temperature, $T_{C}$, is only $\sim 100 \mathrm{~K}^{1,2}$. In addition, recent theoretical and experimental studies have shown that bulk BMO has a centrosymmetric monoclinic structure $(C 2 / c)$ which means the ground state of $\mathrm{BMO}$ is not ferroelectric $(\mathrm{FE})^{3}$. Solovyev et al. proposed that hidden antiferromagnetic (AFM) ordering is responsible for the ferroelectricity ${ }^{4}$. These studies have shown the potential of BMO to be a multiferroic, but the combination of strong FM and FE are difficult to achieve.

The magnetic behaviour of BMO is complex owing to competition between FM and AFM coupling. The $6 s^{2}$ lone pair character of $\mathrm{Bi}^{3+}$ results in a much higher Jahn-Teller $(J T)$ structural distortion than, for example, in A-type AFM LaMnO $3(\mathrm{LMO})^{5-10}$. According to the Goodenough-Kanamori rules, this distortion breaks the degeneracy of the $e_{g}$ orbitals as shown in Fig. 1a. The half-filled $d_{z^{2}}\left(d_{3 z^{2}-r^{2}}\right)$ orbitals point towards the empty $d_{x^{2}-y^{2}}\left(d_{3 x^{2}-r^{2}} / d_{3 y^{2}-r^{2}}\right)$ orbitals on the next manganese and such interactions are ferromagnetic. In addition, the superexchange interaction between $\mathrm{Mn}^{3+}$ ions becomes antiferromagnetic when both $e_{g}$ orbitals point perpendicular to the bond direction despite the interaction between $d_{z^{2}}$ and $d_{x^{2}-y^{2}}$ orbitals. On the other hand, the ferromagnetic interaction occurs if one of the $e_{g}$ orbitals points along the bond direction ${ }^{9}$. In $\mathrm{BMO}$, the interaction between the $d_{z^{2}}$ and $d_{x^{2}-y^{2}}$ orbitals happens along both in-plane and out-of-plane directions and leads to three-dimensional (3D) ferromagnetic interactions (see Fig. 1b). However, there are also AFM interactions along the ab-plane (in-plane) direction ${ }^{7-10}$. The special $e_{g}$ orbital ordering in $\mathrm{BMO}$ is caused by the $6 s^{2}$ lone pair character of $\mathrm{Bi}^{3+}$ that leads to $A$ cations displacements along the $<111>$ direction ${ }^{10}$. The $3 \mathrm{D}$ magnetic interactions in BMO are very different to LMO where ferromagnetic planes alternate antiferromagnetically along out-of-plane (A-type AFM). 
(a)



(b)

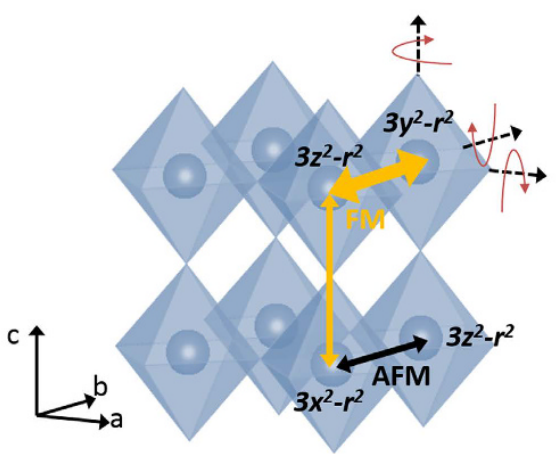

Figure 1. (a) Splitting in energy of the $d$-orbitals of $\mathrm{Mn}^{3+}$ due to the $\mathrm{MnO}_{6}$ octahedron and the further JahnTeller distortion (b) Schematic illustration of the magnetic interactions of $\mathrm{Mn}^{3+}$ ions (blue balls) with $e_{g}$ orbitals within $\mathrm{MnO}_{6}$ octahedra in $\mathrm{BiMnO}_{3}$. The $\mathrm{Bi}^{3+}$ ions at the $A$-site are not shown. Oxygen is at the corners of the octahedra. Yellow arrows and black arrow indicate ferromagnetic and antiferromagnetic superexchange interactions, respectively. The thickness of arrows indicates the strength of the couplings ${ }^{9}$. Red arrows show $\mathrm{MnO}_{6}$ octahedral rotations.

Strain in the structure (whether induced isostatic pressure applied to bulk samples or biaxial pressure applied in thin films by heteroepitaxial growth) can cause octahedral rotations that change the Mn-O-Mn bond lengths and angles. The 3D magnetic state of BMO is very sensitive to changes in $\mathrm{Mn}-\mathrm{O}-\mathrm{Mn}$ bond lengths and angles. Even as low as a $\sim 1^{\circ}$ bond angle change radically alters the magnetic properties ${ }^{7}$. Overall, long-range FM coupling dominates over AFM coupling, but 'hidden' AFM remains and is revealed under certain strain conditions ${ }^{7,8,11}$. This explains why $T_{C}$ s of between 50 and $100 \mathrm{~K}$ have been observed when films are grown on different substrates $^{12-14}$. Therefore, precise tuning of the $\mathrm{Mn}-\mathrm{O}-\mathrm{Mn}$ bond lengths and angles is critical for engineering the FM properties. It should be noted, however, that when the FM properties are optimised, the FE properties are not necessarily optimised ${ }^{1,11,13,15-17}$.

Recently, the improved multiferroic properties of BMO thin films by independently tuning the in-plane and out-of-plane bond lengths, using a combination of isostatic chemical pressure and biaxial mechanical pressure were demonstrated. The chemical pressure was induced by low-level Sm doping of the $A$-site to decrease the overall cell volume and the biaxial mechanical pressure was induced using epitaxial strain. The contraction of the $\mathrm{BiMnO}_{3}$ lattice with $\mathrm{Sm}$, together with the in-plane lattice extension by growth on $\mathrm{SrTiO}_{3}(a=3.905 \AA)$ enabled optimization of both the in-plane and out-of-plane lattice dimensions. An enhancement of FM interactions in the out-of-plane direction by reducing the out-of-plane lattice parameter and a reduction of AFM interactions in-plane by increasing the in-plane lattice parameter were shown. An increase in the $T_{C}$ to $140 \mathrm{~K}$ was achieved, as well as FE behaviour at room temperature (RT). However, since the aim of the earlier work was to measure FE properties, the films were grown relatively thick $(\sim 200 \mathrm{~nm})$, in order for the FE leakage to be minimised, and so uniform straining of the $\mathrm{Bi}_{1-\mathrm{x}} \mathrm{Sm}_{\mathrm{x}} \mathrm{MnO}_{3}(\mathrm{x}=0.15, \mathrm{BSMO})$ film was lost. Consequently, a range of strain states in the films meant that large AFM component remained in the films and a strong tail behaviour in the magnetic transition was observed together with an AFM transition at $\sim 30 \mathrm{~K}$, indicating the competition between the AFM and FM interactions $s^{9,11,13,18,19}$. This result is in agreement with the suggestion that hidden AFM ordering is responsible in FE materials ${ }^{4}$.

The main aim of this work is to achieve a range of different strain states in BSMO thin films so as to determine the conditions for optimising its magnetic properties, i.e. to minimise the AFM component and maximise the FM component. A secondary aim is to shed light on the magnetic phase diagram. The ferroelectric properties of the very thin films of this work were not measured. This is because thin film oxide perovskite ferroelectrics (no matter their composition) show leaky behaviour, and the $20 \mathrm{~nm}$ BSMO films studied here are no exception. Also, the bottom electrode, $\mathrm{SrRuO}_{3}$, which would need to be used on the different insulating substrates, may induce unexpected octahedral rotations in the BSMO films ${ }^{20}$. On the other hand, we have already demonstrated that relatively thick BSMO films grown on $\mathrm{SrTiO}_{3}$ show excellent ferroelectric properties at $\mathrm{RT}^{18}$. Further work 


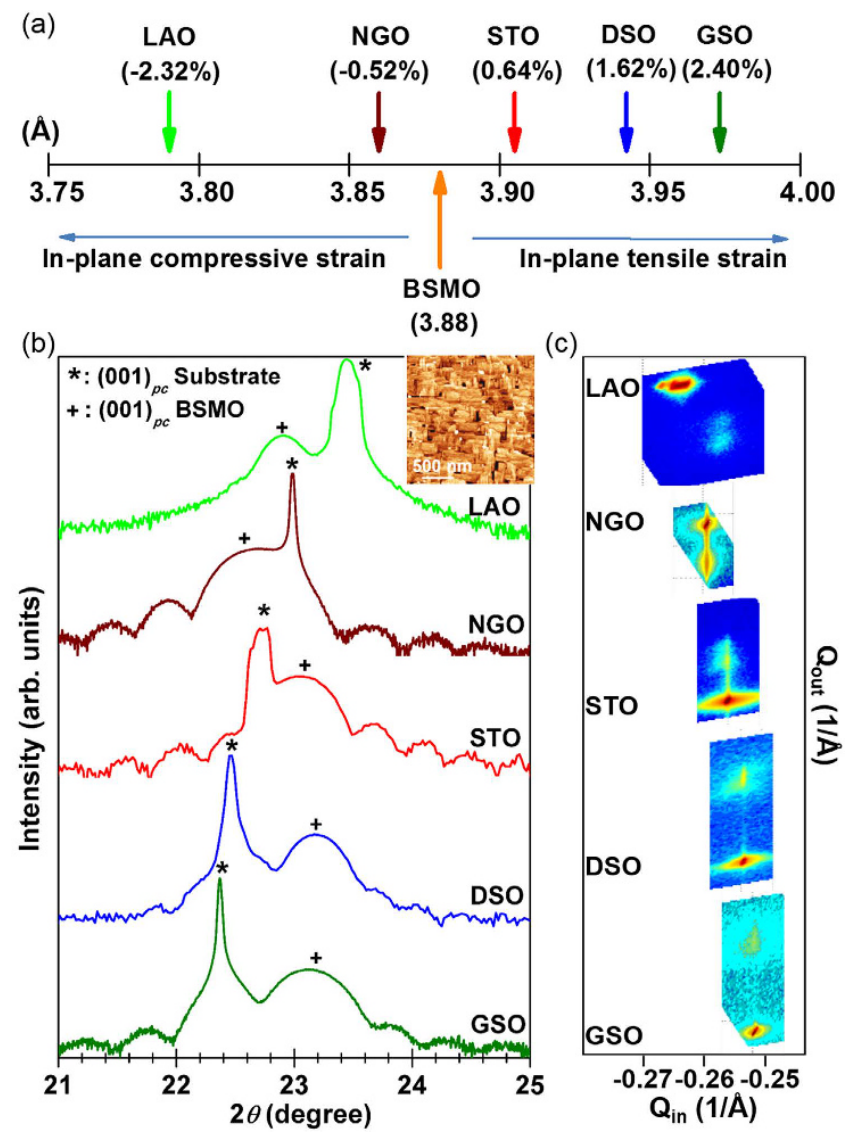

Figure 2. (a) Lattice mismatch between BSMO and the various substrates at RT. XRD spectra of $\sim 20 \mathrm{~nm}$ thick BSMO films grown on the various substrates: (b) The BSMO (001) peak and substrate peak are marked by $(+)$ and $\left({ }^{*}\right)$, respectively. Reflections are indexed based on pseudo-cubic symmetry. The inset shows an atomic force microscopy image with $2 \times 2 \mu \mathrm{m}^{2}$ for BSMO grown on STO. The root-mean-square (RMS) roughness of the film was $\sim 0.26 \mathrm{~nm}$. (c) RSMs around the (332) peak of DSO, GSO and NGO, and (103) of STO and LAO. The substrates peaks are the sharper, red-centred, high intensity peaks which appear at the bottom of the RSM plots, except for LAO and NGO.

is required to measure the $\mathrm{FE}$ properties without changing the octahedral rotations from $\mathrm{SrRuO}_{3}$ (or any other bottom electrode film), for example by using second-harmonic generation studies of films grown directly on insulating substrates.

Very thin films $(\sim 20 \mathrm{~nm})$ of BSMO were grown on a range of different substrates. It was necesssary to grown very thin films $(\sim 20 \mathrm{~nm})$ to provide a near-uniform strain state. The structural distortion of the films was measured using conventional $\mathrm{x}$-ray diffraction (XRD). This gives an indirect assessment of the octahedral rotations: the further the (pseudo-) tetragonal distortion $(c / a)$ is from 1 , the greater level of rotation ${ }^{20-23}$. While synchrotron XRD would be optimum to determine bond angles ${ }^{21,22}$, these measurements were not performed here since the aim of work to demonstrate property enhancement though careful materials engineering.

The key finding of our work is that by engineering an optimal structural distortion into BSMO films, enhanced FM $T_{C}$ 's with clear transitions, up to $176 \mathrm{~K}$, can be achieved. At the same time, by engineering a range of structural distortions into films, the magnetic phase diagram was determined.

Two different experiments were undertaken. The first one explored the magnetic properties of the films of constant thickness $(20-24 \mathrm{~nm})$ on substrates with different lattice mismatch. Thicknesses of $\sim 20 \mathrm{~nm}$ were low enough to give coherent growth with little relaxation of strain but thick enough to give a sizeable magnetic signal. The second study focused on the magnetic properties of films of a range of thicknesses $(10,20$ and $200 \mathrm{~nm})$ grown on $\mathrm{SrTiO}_{3}$ (STO). Films were grown on orthorhombic, paramagnetic (110) $\mathrm{DyScO}_{3}$ (DSO), (110) $\mathrm{GdScO}_{3}$ (GSO), (110) $\mathrm{NdGaO}_{3}$ (NGO) substrates, on cubic, non-magnetic (001) $\mathrm{TiO}_{2}$-terminated STO, and on rhombohedral, non-magnetic (001) $\mathrm{LaAlO}_{3}(\mathrm{LAO})$ substrates. All substrates were thermally treated to show well defined terraces before deposition. The lattice mismatch values between the BSMO and the various substrates are shown in Fig. $2 \mathrm{a}$. BSMO lattice parameter is assumed from a pseudo-cubic cell volume of $58.8 \AA^{318,24,25}$.

\section{Results and Discussion}

Figure $2 \mathrm{~b}$ shows $2 \theta-\omega \mathrm{XRD}$ patterns of $20-24 \mathrm{~nm}$ BSMO films grown on the various substrates. The films were fully epitaxial and no impurity phases were observed (Fig. 2b). Clear thickness fringes for all these films were observed (except for LAO), indicating the high crystal quality. From NGO to GSO, the (001) pc peak of the BSMO 


\begin{tabular}{|c|c|c|c|c|c|c|c|c|c|}
\hline Substrate & \begin{tabular}{|c|} 
In-plane lattice \\
parameter \\
Substrate $[\AA]$ \\
\end{tabular} & $\begin{array}{c}\text { Lattice } \\
\text { mismatch (\%) }\end{array}$ & $\begin{array}{c}\text { In-plane lattice } \\
\text { parameter } \\
\text { BSMO }(\AA)\end{array}$ & \begin{tabular}{|c|} 
Out-of-plane \\
lattice parameter \\
BSMO $[\AA]$
\end{tabular} & \begin{tabular}{|c|} 
Pseudo- \\
cubic unit cell \\
volume $\left[\AA^{3}\right]$
\end{tabular} & \begin{tabular}{|c|} 
(Pseudo-) \\
Tetragonal \\
distortion, $c / a$ \\
\end{tabular} & $\begin{array}{l}\text { Crystal } \\
\text { Structure } \\
\text { BSMO }\end{array}$ & $T_{C}(\mathbf{K})$ & $\begin{array}{c}M r\left(\mu_{\mathrm{B}} / \mathrm{Mn}\right) \\
\text { at } 10 \mathrm{~K}\end{array}$ \\
\hline $\mathrm{LAO}(001)$ & 3.790 & -2.32 & $3.89 \pm 0.005$ & $3.88 \pm 0.005$ & $58.7 \pm 0.2$ & $0.997 \pm 0.005$ & Tetragonal & $95 \pm 10$ & 0.10 \\
\hline $\mathrm{NGO}(110)^{\mathrm{a}}$ & 3.864 & -0.52 & $3.86 \pm 0.005$ & $3.93 \pm 0.005$ & $58.6 \pm 0.2$ & $1.018 \pm 0.005$ & Orthorhombic & $100 \pm 6$ & 0.11 \\
\hline STO (001) & 3.905 & 0.64 & $3.90 \pm 0.005$ & $3.845 \pm 0.005$ & $58.6 \pm 0.2$ & $0.984 \pm 0.005$ & Tetragonal & $150 \pm 3$ & 0.12 \\
\hline $\operatorname{DSO}(110)^{\mathrm{a}}$ & 3.943 & 1.62 & $3.94 \pm 0.005$ & $3.81 \pm 0.005$ & $59.1 \pm 0.2$ & $0.967 \pm 0.005$ & Orthorhombic & $160 \pm 3$ & 0.18 \\
\hline $\mathrm{GSO}(110)^{\mathrm{a}}$ & 3.973 & 2.40 & $3.97 \pm 0.005$ & $3.83 \pm 0.005$ & $60.4 \pm 0.2$ & $0.965 \pm 0.005$ & Orthorhombic & $83 \pm 3$ & 0.06 \\
\hline
\end{tabular}

Table 1. Pseudo-cubic unit cell lattice parameters, lattice mismatch, unit cell volume, (pseudo-) tetragonal distortion, crystal structure, magnetic transition temperature $\left(T_{C}\right)$ and remanent magnetic

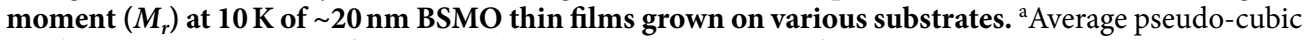
in-plane lattice parameter of orthorhombic structure determined from the RSMs.

shifts to a higher $2 \theta$ angle indicating a reduction of the out-of-plane lattice parameter, which is consistent with the in-plane strain becoming more tensile across the series, as expected. The inset of Fig. 2b shows an AFM height image of a typical film grown on STO. A homogeneous surface morphology of aligned rectangular faceted grains was observed.

Figure 2c shows reciprocal space maps (RSMs) around the (332) peak of DSO, GSO and NGO, and (103) of STO and LAO. The coincidence of the substrate and film in-plane reciprocal lattice vector values $\left(Q_{\text {in }}\right)$ from NGO to GSO indicates that the films are fully in-plane strained to the substrates. The shift of the film peaks to higher $\mathrm{Q}_{\mathrm{in}}$ values indicates an increase of the in-plane lattice parameter which is expected as the substrate lattice parameter increases (Fig. 2c). On the other hand, consistent with Fig. 2b, the out-of-plane reciprocal lattice vector $\left(\mathrm{Q}_{\text {out }}\right)$ shifts to higher values as the substrate lattice parameters increase. The film on LAO is fully relaxed, showing that the growth is incoherent for such large in-plane compressive substrate strain $(-2.32 \%)$. This also explains the absence of thickness fringes in the out-of-plane spectra for BSMO on LAO (Fig. 2b).

A summary of lattice parameters, cell volumes, (pseudo-)tetragonal distortions (the ratio between out-of-plane and in-plane lattice parameters, $c / a)$, crystal structures, FM transition temperatures $\left(T_{C}\right)$ and remanent magnetic moment $\left(M_{r}\right)$ of the $\sim 20 \mathrm{~nm}$ BSMO films are shown in Table 1 .

For all the films, the BSMO cell volume is significantly reduced compared to the bulk BMO value of $61.5 \AA^{3}$. This is consistent with the partial replacement of $\mathrm{Bi}^{3+}$ by the smaller $\mathrm{Sm}^{3+}$ ion ${ }^{18}$. Except for BSMO on GSO $\left(60.4 \pm 0.2 \AA^{3}\right)$, the pseudo-cubic cell volume of all the BSMO films was around $58.8 \pm 0.3 \AA^{3}$. The increase in unit-cell volume on GSO is likely due to the large biaxial tensile in-plane strain $(+2.4 \%)$, which cannot be compensated for by elastically reducing the out-of-plane lattice parameter to such a large extent, and so instead other out-of-plane strain relaxation mechanisms must come into play, e.g. compositional modification such as oxygen loss or cation non-stoichiometry. This is in contrast with BSMO on LAO. Here, there is a similar strain level but it is of opposite sign (-2.32\%). Unlike BSMO on LAO, GSO can maintain in-plane straining because it is possible to strengthen the $\mathrm{Mn}-\mathrm{O}-\mathrm{Mn}$ bonds. On the other hand, for the films on LAO, it is not possible to decrease the $<$ $\mathrm{Mn}-\mathrm{O}-\mathrm{Mn}\rangle$ angle sufficiently to accommodate the in-plane strain.

For NGO, STO and DSO substrates which produce more moderate in-plane strain, $c f$. LAO or GSO, the out-of-plane lattice parameters are increasingly reduced across the series, as the in-plane lattice parameter is increased. This is as expected from simple elastic deformation arguments. The crystal structures of the BSMO films were determined from the asymmetric RSMs at various phi-angles (See Fig. 2c, and Supporting Information). The films on STO and LAO were found to be tetragonal, while the films on DSO, GSO and NGO had an orthorhombic-like structure.

To investigate the effect of biaxial strain on the magnetic properties of the BSMO films, field cooled $M-T$ measurements were undertaken. Herein, normalised $M-T$ curves are shown because these are measured under two different conditions: BSMO on STO and LAO were measured at a field of 200 Oe whereas $M-T$ curves of BSMO on DSO, GSO and NGO were measured at 0 Oe after field cooling at $1 \mathrm{~T}$. The two conditions were necessary because of very high paramagnetic background of DSO, GSO and NGO substrates. For the same reason, magnetic hysteresis curves are not shown. Instead, in Table $1, M_{r}$ at $10 \mathrm{~K}$ is shown for the different films.

Figure 3 shows the $M-T$ curves of the BSMO films on the various substrates. The data can be separated into two different types of curve. The first type shows a tail behaviour. This is found for BSMO on LAO and NGO. Such a behaviour has been reported before for BMO films and indicates the competition between AFM and FM interactions $s^{9,11,13,19}$. When the AFM interactions become dominant, the tail behaviour becomes stronger ${ }^{19}$. In addition to the tail behaviour, the BSMO films on LAO and NGO substrates also show clear AFM transitions at $\sim 30 \mathrm{~K}$, which means the hidden AFM is revealed at low temperature ${ }^{18}$.

The second type of curve shows a clear FM transition and no tail behaviour. This was the case for BSMO on STO, DSO and GSO. Here, $T_{C}$ s ranged from $80 \mathrm{~K}$ on GSO to $160 \mathrm{~K}$ on DSO, the latter value being enhanced by $60 \mathrm{~K}$ compared to strained BMO films ${ }^{13,14}$. For BSMO on DSO and GSO, the magnetic moments increase at low temperature in spite of the clear FM transitions being observed. This is not consistent with a standard FM feature where the magnetic moment saturates at low temperature. The results indicate the presence of AFM coupling ${ }^{26-28}$. For BSMO on STO, a ferromagnetic transition at $150 \mathrm{~K}$ is observed with a clear AFM transition at $\sim 30 \mathrm{~K}$ also observed. The explanation of these two different magnetic behaviours is discussed later.

To understand the relation between magnetic behaviour and strain, $T_{C}$ and $T_{N}$ are plotted versus the (pseudo-) tetragonal distortion $(c / a)$, as shown in Fig. 4 . A clear and very sensitive relationship between the magnetic behaviour of BSMO and the (pseudo-) tetragonality of the unit cell is observed. First of all, for $c / a<0.99$, a clear FM 


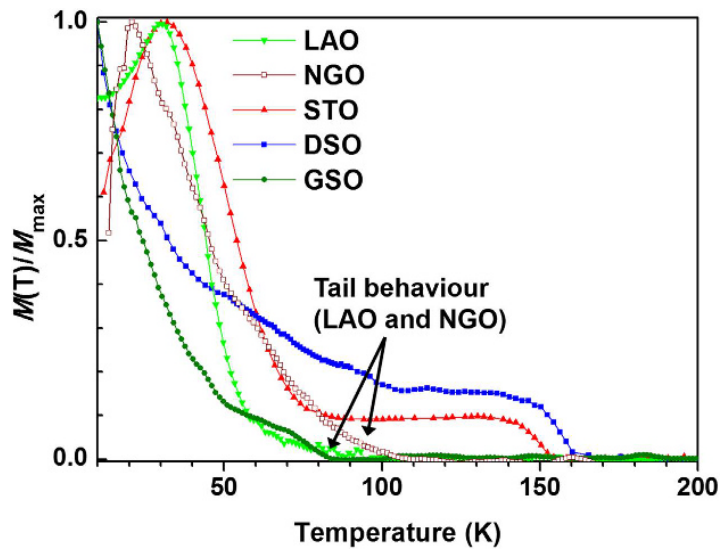

Figure 3. The temperature dependence of magnetisation $(M-T)$ for $\sim 20 \mathrm{~nm}$ BSMO films grown on various substrates. The normalized in-plane $M-T$ curves of BSMO on STO and LAO were measured at a field of 200 Oe. The $M-T$ curves of BSMO on DSO, GSO and NGO were measured at 0 Oe after field cooling at $1 \mathrm{~T}$. Arrows indicate the tail behaviour present for LAO and NGO substrates.

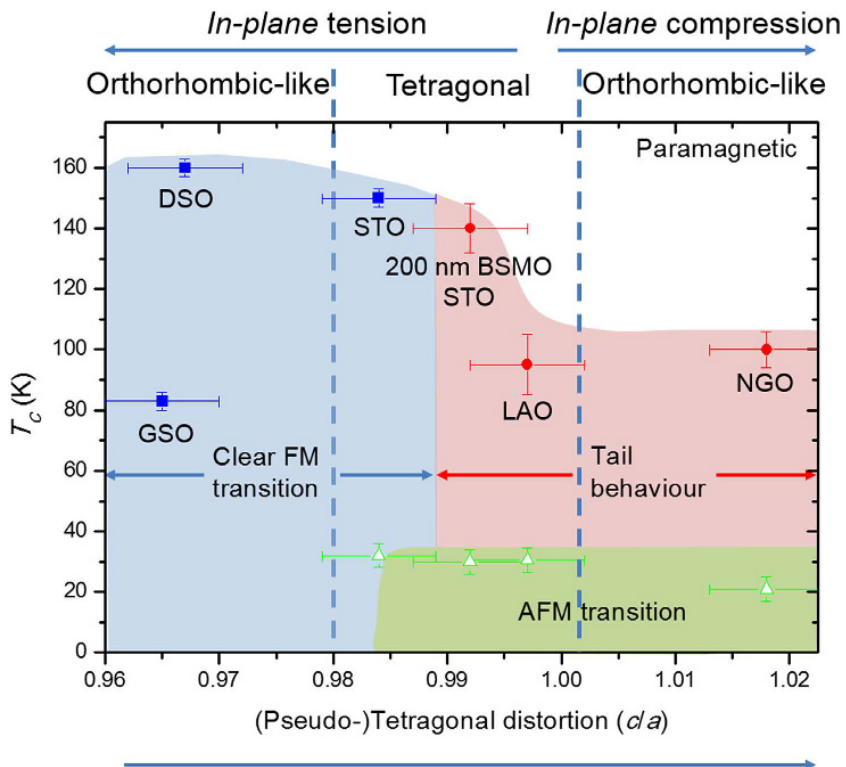

Reduce in-plane $<\mathrm{Mn}-\mathrm{O}-\mathrm{Mn}>$ bonding angle

Figure 4. Magnetic phase diagram for BSMO showing the relationship between tetragonality of pseudocubic and the magnetic transition temperature based on $\sim 20 \mathrm{~nm}$ thick BSMO films studied in this paper. The data for $200 \mathrm{~nm}$ thick BSMO on STO (from ref. 18) is included to provide more information about the transition region near $c / a \sim 0.99$.

transition without tail behaviour is obtained. For $c / a>0.99$, hidden AFM is obtained and is manifest as a tail behaviour in the $M-T$ curves. The strong relationship between magnetic structure and $c / a$ is consistent with other manganites ${ }^{26,29-33}$. For example, for $\mathrm{La}_{0.5} \mathrm{Sr}_{0.5} \mathrm{MnO}_{3}$, by varying the $c / a$ ratio from 0.97 to 1.06 , the magnetic phase changes from A-type AFM to FM to C-type $\mathrm{AFM}^{29}$. According to recent theoretical calculations on $\mathrm{LaMnO}_{3}$, an insulating FM phase is formed under small tensile strain whereas a metallic FM phase and an A-type AFM phase is formed under large compressive and tensile strain, respectively ${ }^{34}$. The sensitivity of the magnetic behaviour with $c / a$ ratio in manganites is caused by the change of the JT distortion of the $\mathrm{MnO}_{6}$ octahedra and the change of the $\mathrm{Mn}-\mathrm{O}-\mathrm{Mn}$ bond lengths and angles.

To understand why $T_{C}$ increases when the $c / a$ ratio is reduced, it is necessary to understand more about the precise crystal structure and the $\mathrm{MnO}_{6}$ octahedral rotations. Minor strained and relaxed BSMO films were found to have a tetragonal crystal structure, indicating either $a^{0} a^{0} c^{-}$or $a^{0} a^{0} c^{+}$octahedral rotation patterns (Glazer's notation $)^{35}$. On the other hand, when the films are grown under large strain, either tensile or compressive, the film crystal structure is orthorhombic-like, as determined by doing x-ray scans around the (103) $)_{\mathrm{pc}}$ peaks along the four pseudo-cubic in-plane directions (see Supporting Information). This indicates that the oxygen octahedra have an $a^{-} a^{-} c^{+}$rotation pattern. 


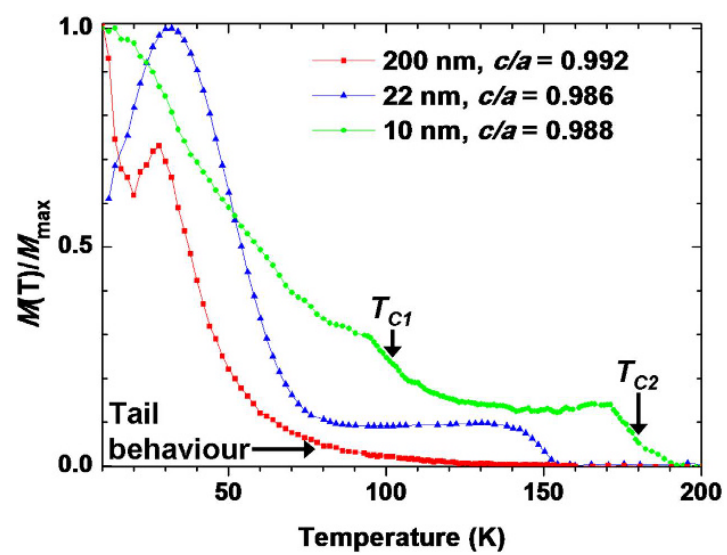

Figure 5. The normalized in-plane $M-T$ curves for BSMO thin films on STO with different thickness, 200 (taken from ref. 18), 20 and $10 \mathrm{~nm}$ at a field of $200 \mathrm{Oe}$.

From previous studies of strained oxide perovskites, $\mathrm{ABO}_{3}$, the degree of rotation along both the in-plane and out-of-plane axes depends strongly on the amount and sign of the strain ${ }^{20,21}$. Hence, under compressive in-plane strain $(c / a>0.98)$, there are strong rotations along the out-of-plane axis, in combination with weaker rotations along the in-plane axis ${ }^{36}$. Under tensile strain $(c / a<0.98)$, it is the other way around: strong rotations occur along the in-plane axes and weaker rotations along the out-of-plane axis. The change in rotation patterns and the degree of the rotations impact the $\langle\mathrm{Mn}-\mathrm{O}-\mathrm{Mn}\rangle$ bond angles and, as a result, the magnetic behaviour.

Taking the above information into account, for strong tensile strained orthorhombic-like BSMO $(c / a<0.98)$, stronger in-plane FM interactions are exepcted (see Fig. 1b) since the in-plane bond angles are likely to be relatively close to $180^{\circ}$, as a result of the weak rotations along the out-of-plane axis.

Looking at the left hand region of Fig. 4 , this is indeed observed, i.e. the high $T_{C}$ samples show clear FM transitions. The results agree with literature, where rare earth manganites and cobaltites show stronger FM interactions and higher $T_{C} \mathrm{~s}$ when the bond angles are close to $180^{\circ} 36,37$.

The highest $T_{C}(\sim 160 \mathrm{~K})$ was found for BSMO on DSO $(c / a=0.967 \pm 0.005)$. A reduced $T_{C}$ of $\sim 83 \mathrm{~K}$ was found for BSMO on GSO. This film would be expected to have the highest $T_{C}$ based on it having the lowest $c / a$ ratio. However, it is very likely that this film has an increased defect concentration because of the very large in-plane strain which results in a significant increase in the unit-cell volume (Table 1). For the films on DSO and GSO, the in-plane FM interaction becomes stronger and dominant, and so the AFM signal becomes relatively weak because the AFM interaction is hidden by the strong FM signal. This is the same situation as in bulk BMO where only FM behaviour is observed. Instead of showing a clear AFM behaviour, i.e. an AFM transition and/or a tail behaviour, the $M-T$ curves for films on DSO and GSO are not saturated at low temperature (see Fig. 3). This lack of saturation is indicated by remanent AFM components, which could originate from a cluster-glass magnetic state ${ }^{28}$.

Even though there is remanent AFM behaviour, the $M_{r}$ of BSMO on DSO has the largest value $\left(\sim 0.18 \mu_{\mathrm{B}} / \mathrm{Mn}\right)$. This is because of a stronger FM behaviour arising from dominant FM interactions. On the other hand, BSMO on GSO has the lowest $M_{r}\left(\sim 0.06 \mu_{\mathrm{B}} / \mathrm{Mn}\right)$, consistent with increased defects in the film.

Looking at the middle region $(0.98<c / a<1)$ of Fig. 4 where there is a low level of in-plane tension, the BSMO is tetragonal instead of orthorhombic-like as was observed for high in-plane tension $(0.98<c / a)$. Since in-plane rotations are absent for this low strain level, there are stronger out-of-plane FM interactions, with an out-of-plane $\langle\mathrm{Mn}-\mathrm{O}-\mathrm{Mn}\rangle$ bond angle of $180^{\circ}$. This leads to an intermediate magnetic state and explains why for STO and LAO AFM interactions (at $\sim 30 \mathrm{~K}$ ) are observed in addition to FM interactions (see Fig. 3).

Finally, looking at the right hand region $(c / a>\sim 1)$ of Fig. 4 where there is a higher lever of in-plane compressive strain, an orthorhombic-like structure is produced once more for BSMO on NGO. Hence, in this region, stronger out-of-plane FM interactions and weaker in-plane FM interactions are expected. Therefore, the in-plane FM interactions do not dominate the magnetic behaviour. As a result, $T_{C}$ decreases as the $c / a$ ratio is increased, and a clear AFM transition is observed around $30 \mathrm{~K}$, as well as an AFM tail behaviour (see Fig. 3).

Finally, by studying different thickness films on STO, the intermediate transition region $(0.980<c / a<0.995)$ of Fig. 4 is probed more closely. Figure 5 shows the $M-T$ curves for BSMO films on STO of 200, 20 and $10 \mathrm{~nm}$ thickness. The different film thicknesses result in different levels of strain relaxation and hence different $c / a$ ratios. For the $200 \mathrm{~nm}$ thick film, the $T_{C}$ is enhanced to $\sim 140 \pm 6 \mathrm{~K}$ ( $c f$. the bulk BMO $T_{C}$ of $\sim 100 \mathrm{~K}$ ). Both a tail behaviour and an AFM transition at $30 \mathrm{~K}$ are observed but not a clear FM transition. For the $20 \mathrm{~nm}$ thick film, a clear FM transition is observed with $T_{C}$ of $150 \pm 3 \mathrm{~K}$. There is no tail behaviour, but there is an AFM transition at $\sim 30 \mathrm{~K}$.

For the $10 \mathrm{~nm}$ thick film, a clear FM transition is observed with $T_{C}$ (termed $T_{C 2}$ ) of $176 \pm 3 \mathrm{~K}$. There is no tail behaviour, nor a $30 \mathrm{~K}$ AFM transition. However, there is a second FM transition with $T_{C}$ (termed $T_{C l}$ ) at $100 \pm 3 \mathrm{~K} . T_{C 2}$ is surprisingly large, enhanced by $\sim 70 \mathrm{~K}$ compared to bulk BMO, while $T_{C 1}$ is very close to the $T_{C}$ of bulk BMO. This feature is similar to ultrathin $\mathrm{La}_{0.67} \mathrm{Sr}_{0.33} \mathrm{MnO}_{3}$ (LSMO) films ( $<10$ unit cells) where a double transition is found with a much enhanced $T_{C}$ at $\sim 560 \mathrm{~K}$, and a second lower transition at $\sim 200 \mathrm{~K}$. Thicker LSMO films ( $>20$ unit cells), on the other hand, show bulk $T_{C} s$ of $\sim 350 \mathrm{~K}^{27}$. The two ferromagnetic phases for the $10 \mathrm{~nm}$ thick BSMO film likely originate from one or more of the possible strain relaxation mechanisms which occur in perovskite films, e.g. phase separation, non-stoichiometry, or deformation of $\mathrm{BO}_{6}$ octahedra ${ }^{38-43}$. 
In conclusion, the relation of magnetic properties and crystal structure in $\mathrm{BiMnO}_{3}$, the most promising multiferroic with a relatively high FM $T_{C}$, was determined for the first time. Sm-doping of the Bi site at a level of $15 \%$ was undertaken to reduce the unit cell volume to enhance FM coupling (3D chemical pressure), and different substrates were used (2D biaxial pressure) to expand or contract the unit cell in-plane. Hence, 3D $+2 \mathrm{D}$ biaxial straining were used together to precisely engineer the $\mathrm{BiMnO}_{3}$ crystal structure, so as to optimise the 3D $\mathrm{Mn}-\mathrm{O}-\mathrm{Mn}$ magnetic coupling. A magnetic phase diagram for the system was mapped out for $0.96<\mathrm{c}<1.02$. It was possible to eliminate the hidden AFM and strongly increase the $T_{C}$ by optimizing the (pseudo-)tetragonal distortion $(c / a)$. For $c / a<0.98$, the films showed a clear FM behaviour. On the other hand, for $c / a>0.98$, hidden AFM was revealed in addition to the FM behaviour.

With appropriate choice of substrate, doping and film thickness, it was possible to strongly increase $T_{C}$. A $10 \mathrm{~nm}$ thick Sm-doped $\mathrm{BiMnO}_{3}$ film grown on STO showed a sharp FM $T_{C}$ of $176 \pm 3 \mathrm{~K}$ and a $20 \mathrm{~nm}$ thick $\mathrm{Sm}$-doped $\mathrm{BiMnO}_{3}$ film grown on DSO showed a sharp FM $T_{C}$ of $160 \pm 3 \mathrm{~K}$. Of course, the ultimate goal for BMO films is to achieve both strong FM and FE, and to do this above room temperature. To achieve this, the right strain state needs to be engineered into films thicker than a few 10's of nm, thereby reducing leakage. A possible future approach is to use nanocomposite films where high strain levels can be induced in thick films $(>100 \mathrm{~nm})$ and where enhanced FM and FE properties have already been demonstrated in other perovskites ${ }^{4}$.

\section{Methods}

Films were grown by pulsed laser deposition (PLD) from a single ceramic target. The growth rate was $0.07-0.08 \AA$ / pulse. For the film growth, the laser pulse rate was $2 \mathrm{~Hz}$ with a laser fluence of $1.3 \mathrm{Jcm}^{-2}$. The oxygen pressure was fixed at 100 mTorr and growth temperature was $650^{\circ} \mathrm{C}$. To confirm the phase purity, crystalline quality and the $3 \mathrm{D}$ strain state of the films at RT, $2 \theta-\omega$ scans and reciprocal space maps (RSMs) were carried out using a Panalytical Empyrean high resolution x-ray diffraction (XRD) system. We determined the out-of-plane lattice parameter by measuring the position of the $(00 l)$ peaks in the $2 \theta-\omega$ scans as well as the (103) pseudo-cubic (pc) peak of the BSMO in the RSMs. The film thicknesses were determined by x-ray reflectivity. To determine the film surface morphology, atomic force microscopy was performed. The temperature dependence of magnetisation $(M-T)$ was determined using a superconducting quantum interference device (SQUID) magnetometer (Quantum Design, MPMS).

\section{References}

1. Moreira dos Santos, A. et al. Evidence for the likely occurrence of magnetoferroelectricity in the simple perovskite, $\mathrm{BiMnO}_{3}$. Solid State Commun. 122, 49-52 (2002)

2. Seshadri, R. \& Hill, N. A. Visualizing the Role of Bi 6s "Lone Pairs" in the Off-Center Distortion in Ferromagnetic BiMnO 3 . Chem. Mater. 13, 2892-2899 (2001).

3. Belik, A. A. Polar and nonpolar phases of $\mathrm{BiMO}_{3}$ : A review. J. Solid State Chem. 195, 32-40 (2012).

4. Solovyev, I. V. \& Pchelkina, Z. V. Magnetic-field control of the electric polarization in $\mathrm{BiMnO}_{3}$. Phys. Rev. B 82, 094425 (2010).

5. Belik, A. A. et al. Origin of the Monoclinic-to-Monoclinic Phase Transition and Evidence for the Centrosymmetric Crystal Structure of $\mathrm{BiMnO}_{3}$. J. Am. Chem. Soc. 129, 971-977 (2007).

6. Pinsard-Gaudart, L. et al. Stability of the Jahn-Teller effect and magnetic study of $\mathrm{LaMnO}_{3}$ under pressure. Phys. Rev. B 64, 064426 (2001).

7. Kozlenko, D. P. et al. Competition between ferromagnetic and antiferromagnetic ground states in multiferroic $\mathrm{BiMnO}_{3}$ at high pressures. Phys. Rev. B 82, 014401 (2010).

8. Atou, T., Chiba, H., Ohoyama, K., Yamaguchi, Y. \& Syono, Y. Structure Determination of Ferromagnetic Perovskite BiMnO 3 . J. Solid State Chem. 145, 639-642 (1999).

9. Yang, C.-H. et al. Orbital ordering and enhanced magnetic frustration of strained $\mathrm{BiMnO}_{3}$ thin films. Europhys. Lett. 74, 348-354 (2006).

10. Moreira Dos Santos, A. et al. Orbital ordering as the determinant for ferromagnetism in biferroic $\mathrm{BiMnO}_{3}$. Phys. Rev. B 66, 064425 (2002).

11. Jeen, H. et al. Growth and characterization of multiferroic $\mathrm{BiMnO}_{3}$ thin films. J. Appl. Phys. 109, 074104 (2011).

12. Son, J. Y., Kim, B. G., Kim, C. H. \& Cho, J. H. Writing polarization bits on the multiferroic $\mathrm{BiMnO}_{3}$ thin film using Kelvin probe force microscope. Appl. Phys. Lett. 84, 4971 (2004).

13. Gajek, M. et al. Spin filtering through ferromagnetic $\mathrm{BiMnO}_{3}$ tunnel barriers. Phys. Rev. B 72, 020406 (2005).

14. De Luca, G. M. et al. Ferromagnetism and ferroelectricity in epitaxial BiMnO $\mathrm{Bl}_{3}$ ultra-thin films. Appl. Phys. Lett. 103, 062902 (2013).

15. Yang, H. et al. Is ferroelectricity in $\mathrm{BiMnO}_{3}$ induced by superlattice? J. Mat. Sci. 43, 3604 (2008).

16. Son, J. Y. \& Shin, Y.-H. Multiferroic $\mathrm{BiMnO}_{3}$ thin films with double SrTiO buffer Appl. Phys. Lett. 93, 062902 (2008).

17. Grizalez, M., Mendoza, G. A. \& Prieto, P. Analysis of multiferroic properties in $\mathrm{BiMnO}_{3}$ thin films. J. Phys. Conf. Ser. 167, 012035 (2009).

18. Choi, E.-M. et al. Ferroelectric Sm-Doped BiMnO 3 Thin Films with Ferromagnetic Transition Temperature Enhanced to 140 K. ACS Appl. Mater. Interfaces 6, 14836 (2014).

19. Hirone, T., Maeda, S., Tsubokawa, I. \& Tsuya, N. On the Magnetic Properties of the System MnSb-CrSb. JPSJ 11, 1083-1087 (1956).

20. Vailionis, A. et al. Misfit strain accommodation in epitaxial $A B \mathrm{O}_{3}$ perovskites: Lattice rotations and lattice modulations. Phys. Rev. B 83, 064101 (2011).

21. May, S. J. et al. Quantifying octahedral rotations in strained perovskite oxide films. Phys. Rev. B 82, 014110 (2010).

22. Rotella, H. et al. Octahedral tilting in strained $\mathrm{LaVO}_{3}$ thin films. Phys. Rev. B 85, 184101 (2012)

23. D. P. Kozlenko, V. P. Glazkov, Z. Jirak \& B. N. Savenko, J. Phys.: Condens. Matter 16, 2381-2394 (2004).

24. Jia, Y. Q. Crystal radii and effective ionic radii of the rare earth ions. J. Solid State Chem. 95, 184-187 (1991).

25. Chiba, H., Atou, T. \& Syono, Y. Magnetic and Electrical Properties of $\mathrm{Bi}_{1-x} \mathrm{Sr}_{x} \mathrm{MnO}_{3}$ : Hole-Doping Effect on Ferromagnetic Perovskite $\mathrm{BiMnO}_{3}$. J. Solid State Chem. 132, 139-143 (1997).

26. Sadoc, A. et al. Large Increase of the Curie Temperature by Orbital Ordering Control. Phys. Rev. Lett. 104, 046804 (2010).

27. Boschker, H. et al. High-Temperature Magnetic Insulating Phase in Ultrathin $\mathrm{La}_{0.67} \mathrm{Sr}_{0.33} \mathrm{MnO}_{3}$ Films. Phys. Rev. Lett. 109, 157207 (2012).

28. Fertman, E. et al. Exchange bias in phase-segregated $\mathrm{Nd}_{2 / 3} \mathrm{Ca}_{1 / 3} \mathrm{MnO}_{3}$ as a function of temperature and cooling magnetic fields. J. Appl. Phys. 115, 203906 (2014).

29. Fang, Z., Solovyev, I. V. \& Terakura, K. Phase Diagram of Tetragonal Manganites. Phys. Rev. Lett. 84, 3169 (2000).

30. Suwardi, A. et al. Turning antiferromagnetic $\mathrm{Sm}_{0.34} \mathrm{Sr}_{0.66} \mathrm{MnO}_{3}$ into a $140 \mathrm{~K}$ ferromagnet using a nanocomposite strain tuning approach. Nanoscale 8, 8083 (2016). 
31. Nanda, B. R. K. \& Satpathy, S. Effects of strain on orbital ordering and magnetism at perovskite oxide interfaces: $\mathrm{LaMnO}_{3} / \mathrm{SrMnO}_{3}$. Phys. Rev. B 78, 054427 (2008).

32. Solovyev, I. V. \& Terakura, K. Spin canting in three-dimensional perovskite manganites. Phys. Rev. B 63, 174425 (2001).

33. Baena, A., Brey, L. \& Calderón, M. J. Effect of strain on the orbital and magnetic ordering of manganite thin films and their interface with an insulator. Phys. Rev. B 83, 064424 (2011).

34. Hou, Y. S., Xiang, H. J. \& Gong, X. G. Intrinsic insulating ferromagnetism in manganese oxide thin films. Phys. Rev. B 89, 064415 (2014).

35. Glazer, A. M. The classification of tilted octahedra in perovskites. Acta Cryst. B 28, 3384 (1972).

36. Fuchs, D. et al. Tuning the magnetic properties of $\mathrm{LaCoO}_{3}$ thin films by epitaxial strain. Phys. Rev. B 77, 014434 (2008).

37. Kajimoto, R. et al. R-Dependence of Spin Exchange Interactions in $\mathrm{RMnO}_{3}(\mathrm{R}=$ Rare-Earth Ions). J. Phys. Soc. Jpn. 74, 2430 (2005).

38. Bibes, M. et al. Nanoscale Multiphase Separation at $\mathrm{La}_{2 / 3} \mathrm{Ca}_{1 / 3} \mathrm{MnO}_{3} / \mathrm{SrTiO}_{3}$ Interfaces. Phys. Rev. Lett. 87, 067210 (2001).

39. Wimbush, S. C. et al. Interfacial Strain-Induced Oxygen Disorder as the Cause of Enhanced Critical Current Density in Superconducting Thin Films. Adv. Funct. Mater. 19, 835-841 (2009).

40. Gazquez, J. et al. Lattice mismatch accommodation via oxygen vacancy ordering in epitaxial $\mathrm{La}_{0.5} \mathrm{Sr}_{0.5} \mathrm{CoO}_{3-\delta}$ thin films. APL Mater. 1, 012105 (2013).

41. Vailionis, A. et al. Symmetry and lattice mismatch induced strain accommodation near and away from correlated perovskite interfaces. Appl. Phys. Lett. 105, 131906 (2014).

42. Tebano, A. et al. Evidence of Orbital Reconstruction at Interfaces in Ultrathin $\mathrm{La}_{0.67} \mathrm{Sr}_{0.33} \mathrm{MnO}_{3}$ Films. Phys. Rev. Lett. 100, 137401 (2009).

43. Lee, J.-S. et al. Hidden Magnetic Configuration in Epitaxial $\mathrm{La}_{1-\mathrm{x}} \mathrm{Sr}_{\mathrm{x}} \mathrm{MnO}_{3}$ Films. Phys. Rev. Lett. 105, 257204 (2010).

44. MacManus-Driscoll, J. L., Suwardi, A. \& Wang, H. Composite epitaxial thin films: A new platform for tuning, probing, and exploiting mesoscale oxides. MRS bulletin 40, 933-942 (2015).

\section{Acknowledgements}

This work was supported by the European Research Council (ERC) (Advanced Investigator grant ERC-2009AdG-247276-NOVOX), the Engineering and Physical Sciences Research Council (EPSRC) (Equipment Account Grant EP/K035282/1) and the Isaac Newton Trust (Minute 13.38(k)).

\section{Author Contributions}

E.M.C., J.E.K. and J.L.D. contributed equally. The manuscript was written through contributions of all authors. All authors have given approval to the final version of the manuscript.

\section{Additional Information}

Supplementary information accompanies this paper at http://www.nature.com/srep

Competing Interests: The authors declare no competing financial interests.

How to cite this article: Choi, E.-M. et al. Strain-tuned enhancement of ferromagnetic $T_{C}$ to $176 \mathrm{~K}$ in Sm-doped $\mathrm{BiMnO}_{3}$ thin films and determination of magnetic phase diagram. Sci. Rep. 7, 43799; doi: 10.1038/srep43799 (2017).

Publisher's note: Springer Nature remains neutral with regard to jurisdictional claims in published maps and institutional affiliations.

(c) (i) This work is licensed under a Creative Commons Attribution 4.0 International License. The images or other third party material in this article are included in the article's Creative Commons license, unless indicated otherwise in the credit line; if the material is not included under the Creative Commons license, users will need to obtain permission from the license holder to reproduce the material. To view a copy of this license, visit http://creativecommons.org/licenses/by/4.0/

(c) The Author(s) 2017 